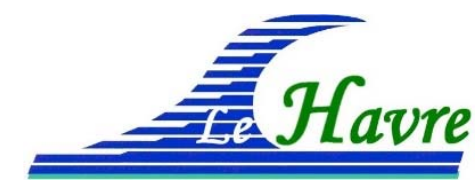

\title{
Hommage à trois voix pour Chantal Bonnot-Courtois, la Dame du Littoral (1949-2019)
}

\author{
Bruno CALINE ${ }^{1}$, Agnès BALTZER ${ }^{2}$, Claire ROLLET ${ }^{3}$
}

1. Ingénieur géologue à la retraite, TOTAL.

2. Université de Nantes, UMR 6554 CNRS - LETG, Campus du Tertre, BP 81 227, 44312 Nantes Cedex 3, France.

agnes.baltzer@univ-nantes.fr

3. Ifremer, Laboratoire Environnement Ressources Bretagne Nord, Station de Dinard, 38 rue du Port Blanc - 35800 Dinard, France.

claire.rollet@ifremer.fr

\section{Résumé :}

Cet article rend hommage à Chantal Bonnot-Courtois, grande sédimentologue du littoral décédée en février 2019 (figure 1). Après une brève biographie, nous avons voulu lui rendre un hommage sincère, exprimé de façon moins académique, par trois collègues et amis.

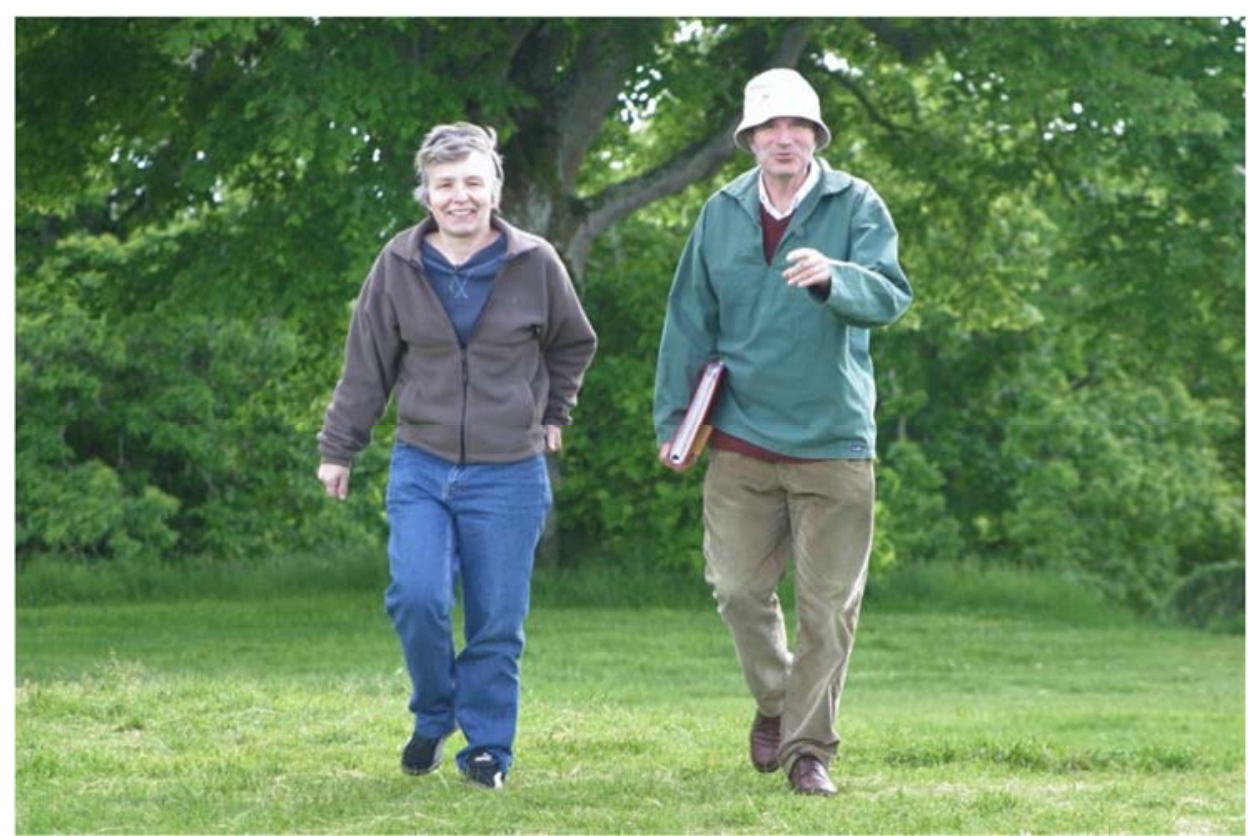

Figure 1. Chantal Bonnot-Courtois et Bruno Caline au Mont-Dol en 2006, (Photo Christian Hubans).

\section{Mots-clés :}

Dame du Littoral, Intertidal, Sédiment, Rigueur, "Gadoue", Vérité terrain, Baie du MontSaint-Michel, Marion Dufresne, Amitié. 


\section{Lecture spéciale}

\section{Biographie courte de La Dame du littoral}

Rien à l'origine ne semblait prédestiner Chantal Bonnot, née Courtois à s'intéresser de près aux rivages de Bretagne et de Normandie. Et pourtant...

"Petite dernière" d'une fratrie de huit, c'est bien loin de la mer, dans les montagnes du Jura que Chantal passait ses vacances étant jeune.

Dès son enfance, elle fut bercée par une évidence à honorer : réussir ses études. Ce qu'elle fit avec brio et devenue étudiante, elle s'orienta vers la géologie à l'université de Paris Sud à Orsay. Avec le titre de Docteur ès sciences, Chantal débuta sa carrière de chercheuse au CNRS à l'université d'Orsay.

Le tournant dans sa carrière se produisit fin 1984 lorsque le couple Bonnot-Courtois et leur 3 jeunes enfants, quitta la région parisienne pour venir poser ses valises à Saint-Briac. Cette jeune chercheuse du CNRS va alors marquer de son empreinte l'équipe de géologues et géographes de ce laboratoire de l'École Pratique des Hautes Études.

Abandonnant la haute mer et les argiles des grands fonds, Chantal concentrera désormais ses travaux de recherche sur la frange littorale en étudiant de près la façon dont les sédiments au cours du temps, naissent, voyagent et finalement se déposent. Cette spécialité de la géologie appelée la sédimentologie est essentielle pour guider les travaux d'aménagement des côtes. Cette discipline n'aura bientôt plus de secret pour Chantal qui étudiera avec soin les rivages de la Manche en Bretagne et Normandie avec une prédilection pour la baie du Mont-Saint-Michel et l'estuaire de la Rance.

Avec efficacité et rigueur tout en restant discrète et modeste, Chantal parcourt alors sur le terrain nombre de plages, baies, îles, falaises, havres, caps, estuaires pour percer le mystère de leur évolution dans le temps à l'échelle géologique mais surtout à l'échelle humaine. Elle combine les observations et mesures au sol avec les images de plus en plus précises fournies par les satellites. Et surtout elle intègre dans son interprétation sédimentologique les données issues des disciplines complémentaires comme la géomorphologie, la biologie, la botanique, l'archéologie et l'histoire. Ces nombreuses missions sur le terrain étaient menées le plus souvent en équipe avec ses collègues biologistes de l'Ifremer (station de Saint-Malo puis de Dinard et Centre Ifremer de Bretagne de Brest), du laboratoire maritime du Muséum national d'Histoire naturelle à Dinard, les sédimentologues de l'université de Caen, du BRGM et de TOTAL, les botanistes de l'université de Rennes et les ethnologues de l'écomusée de la baie du MontSaint-Michel. C'est cette approche pluridisciplinaire qui lui permet de comprendre les interactions entre les phénomènes naturels et les activités humaines afin de recommander les aménagements les mieux adaptés. Grâce à cette démarche, elle devient rapidement une spécialiste en sédimentologie appliquée à l'aménagement des côtes bretonnes et normandes. 


\section{XVIèmes Journées Nationales Génie Côtier - Génie Civil \\ Le Havre 2020}

Ainsi, au cours de sa carrière à Dinard, Chantal aura successivement étudié la dynamique sédimentaire :

- des havres de la façade occidentale du Cotentin (1985-1986),

- de la baie de Saint-Brieuc (1986-1988 puis 1996-1998),

- de l'estuaire de la Rance (1990-1995),

- de la baie du Mont-Saint-Michel (en continu entre 1990 et 2011),

- du domaine marin côtier entre Paimpol, le Cap Fréhel, Saint-Malo et Granville (1996-2006),

- des îles Chausey (2007-2009).

L'impressionnante bibliographie de ses travaux à Dinard est composée de plus de 50 références constituées d'articles scientifiques, de rapports techniques, de cartes thématiques et d'ouvrages de synthèse. Parmi ceux-ci, des références, considérées par la communauté scientifique comme étant incontournables sont données en fin de cet hommage.

Chantal a également participé à de nombreux congrès, symposium et colloques nationaux et internationaux au cours desquels elle soulignait dans ses présentations orales et les excursions sur le terrain la particularité des rivages du golfe Normand-Breton soumis aux marées parmi les plus importantes sur terre. Elle était d'ailleurs "Membre d'honneur" du Centre Français du Littoral Français depuis 2004.

Un grand nombre d'étudiants des universités de Paris Sud (Orsay), de Rouen-Le Havre et Caen ont pu bénéficier des connaissances de Chantal sur le domaine littoral car en parallèle à ses travaux de recherche, elle a régulièrement animé au laboratoire de Géomorphologie de Dinard (situé boulevard de la Mer) des cours et stages en donnant toujours la priorité à l'observation directe sur le terrain des sédiments "vivants" ce qui facilite l'interprétation des processus physiques et biologiques responsables de leur formation et de leur évolution dans le temps.

Au début de sa carrière, rien ne semblait donc prédestiner Chantal (qui n'avait pas le pied marin!) à se tourner vers l'étude des rivages de la Manche. Et pourtant.... la suite a montré que cette scientifique, directrice de recherche au CNRS, animée par la curiosité, la volonté et la rigueur est devenue une experte en sédimentologie appliquée au littoral.

Elle laissera un grand vide autour d'elle car ses anciens collègues et collaborateurs appréciaient ses qualités humaines, son humour, sa franchise et son écoute. Pour nombre d'entre eux, Chantal était devenue une amie qui leur sera impossible d'oublier.

\section{Témoignages de Claire et Agnès}

\subsection{Témoignage de Claire}

Agnès, tu m'as appelée m'invitant à partager ce texte, hommage à notre amie, cette si belle personne. Alors je dis 'Oui !' et je propose ici défiant toute convention, quelques moments précis qui ont marqués nos vies auprès de cette dame, La Dame du Littoral. 


\section{Lecture spéciale}

Nous croiserons ici quelques beaux souvenirs et saurons rendre hommage à cette grande Dame des sciences du littoral, Chantal Bonnot-Courtois que nous aimions tant. Nous étions des collègues qui parfois, et même très souvent, nous retrouvions si justement en phase pour échanger encore, pour partager des fruits de mers, pour finir un article, pour goûter le comté ramené du Jura...

Nous égrènerons quelques-unes de ses publications. Mais faut-il tout citer ? Je ne le pense pas. Les outils numériques bien choisis devraient suffirent, encore que trop souvent elle rappelait combien tous ces jeunes chercheurs oubliaient sans scrupules les travaux des scientifiques du passé. Et cela la fâchait. Il en fallait pourtant beaucoup pour la contrarier. Quelle belle rencontre ! C'était en 1989, jeune étudiante je venais m'enquérir auprès de spécialistes de la Rance de connaissances pouvant m'aider à la rédaction d'une synthèse de la situation de l'aquaculture en Rance et de son évolution. Et oui, je ne suis pas géologue, seulement biologiste... C'est une dizaine d'années plus tard, au retour d'un moment de ma vie passée au Québec que je retrouve Chantal. Un projet de cartographie des habitats benthiques sur le pourtour breton. Chantal était l'experte sédimentologue du littoral, j'étais la biologiste-cartographe de tout l'estran breton. Nous avons écumé, ensemble, à pied, droites dans nos bottes, près de $170 \mathrm{~km}^{2}$ d'estran au cours d'une trentaine de campagnes de validation terrain, soit plus de 90 marées de vives eaux... par tous les temps. Nous étions toujours prêtes... Nous rentrions parfois le nez brûlé par le soleil, d'autres fois détrempées par la pluie battante.

Cette vérité terrain qui lui était si chère, savoir observer les sédiments "vivants", y goûter presque parfois pour nous amuser, afin de mieux comprendre les processus physiques, biologiques aussi. Nous croisions nos regards sur cette diversité. De longues discussions, et son regard expert sur les mouvements de sables, des vases au fil du temps. De cette lecture si fine du paysage côtier, j'ai pu appréhender le littoral dans toutes ses dynamiques, ses libertés aussi. Ce littoral dompté, endigué, empierré pour l'usage de l'homme et qui souvent encore se rebelle les jours de grande tempête pour retrouver son droit, une liberté mouvante dénoncée, assumée.

Sur les côtes bretonnes et normandes, elle était une experte à chaque fois consultée pour tout aménagement du littoral. Combien de cartes, combien de cycles de marée a-t-elle dessinés, avec extrême justesse, précise jusque dans les moindres détails, pointilleuse sur l'exactitude des mots, exigeante aussi bien sur le fond que la forme. Et c'est ainsi que certaines figures de variations de niveaux de marées pouvaient ressembler à des ailes de papillon dans la Baie du Mont-Saint-Michel (figure 2)... Cette Baie du Mont-SaintMichel qu'elle a si souvent sillonnée. 


\section{XVİ̀mes Journées Nationales Génie Côtier - Génie Civil \\ Le Havre 2020}

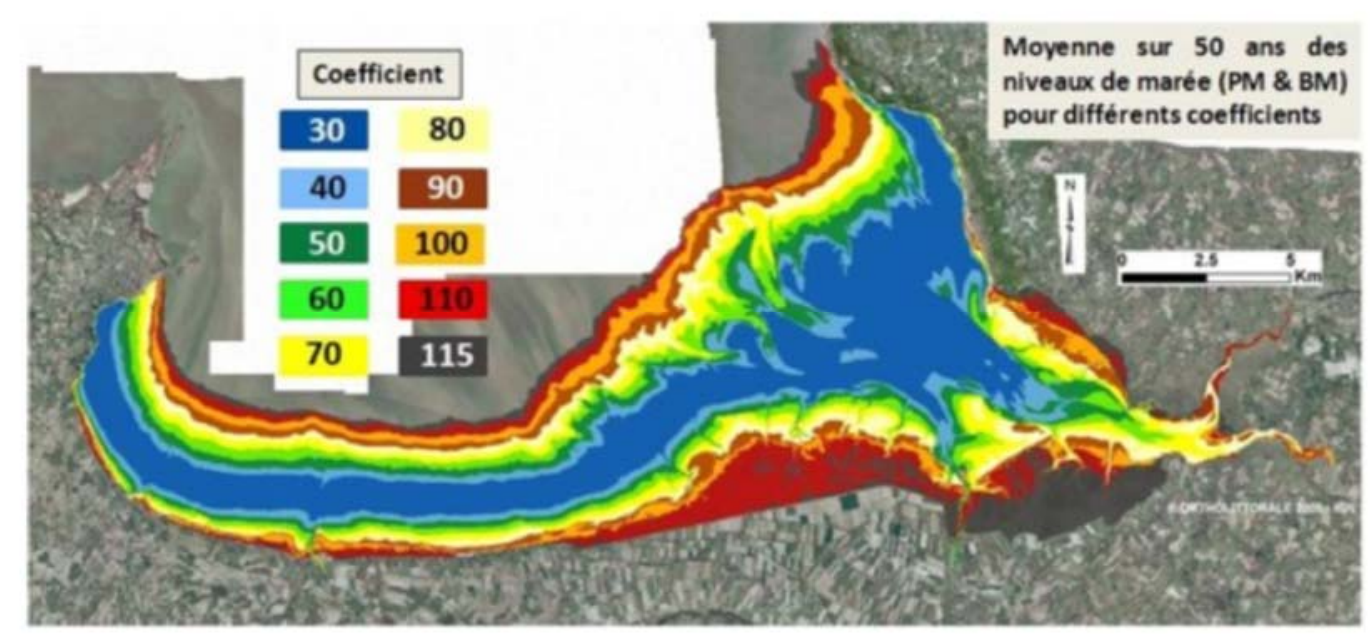

Figure 2. Balayage de la zone intertidale par les marées des différents coefficients classés de 10 en 10. Moyennes des niveaux de Pleine Mer et Basse Mer, établies sur 50 ans (1950 à 2000), d'après Bonnot-Courtois et al., 2012.

\subsection{Témoignage d'Agnès}

Oh oui, je me souviens bien de cette Baie du Mont-Saint-Michel! En effet, fraîchement arrivée à l'Université de Caen, déboulant de mon post-doc en Écosse sur les avalanches sous-marines, je ne connaissais rien au domaine littoral et je m'étais glissée dans un stage de Géomorphologie et Aménagement du Littoral, piloté par Mme Bonnot-Courtois et M. Levacher.

A peine avais-je mis un pied sur l'estran, qu'il me fallait traîner une luge chargée de tout le matériel scientifique, avec Monique et Bernadette, pour faire les trois fameux transects de plusieurs kilomètres chacun, de Vildé-Hirel, de Cherrueix et de Cancale, chaque mois (figure 3), lors des marées de vives eaux dans le cadre du Chantier PNEC (Programme National Environnement Côtier). Au bout de quelques-unes de ces longues sorties de terrain, chacun d'entre nous essayait de trouver une bonne raison de ne pas être aux environs du Laboratoire de Dinard par les jours de grande marée. Et pourtant, à chaque fois, malgré les efforts à fournir, ces moments de terrain partagés dans la vase, la 'gadoue' restent ancrés comme de précieux souvenirs.

Mais plus loin, entre Erquy et Fréhel, que de révélations sur la plage des Sables d'Or ! Que de grains de sables retournés, de rides sableuses décortiquées et de deltas de flots discutés.... Et toujours, toujours, elle répétait : "Regarde l'avant côte... ", "Regarde là ! Juste sous la mer, c'est là que se jouent la formation des plages et les transformations du littoral...". 


\section{Lecture spéciale}
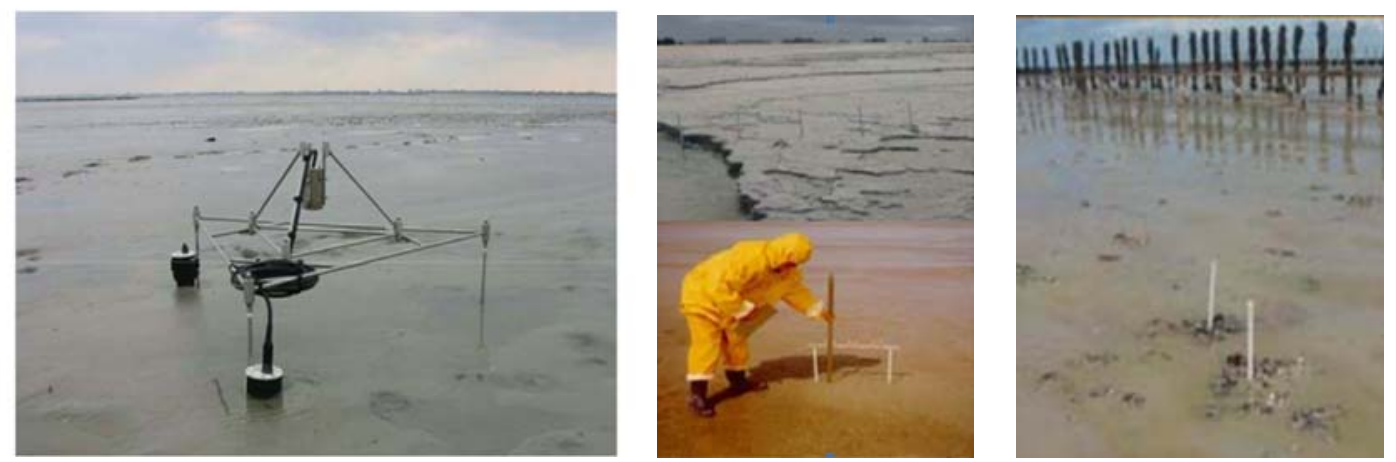

Figure 3. Dispositif ALTUS (photo de gauche) et stations d'implantation de jalons (photos milieu et à droite) pour le suivi moyen terme du niveau du sédiment, d'après Bonnot-Courtois et al., 2006.

Car la Dame savait aussi la mer... Elle avait commencé sa carrière dans les prestigieux laboratoires parisiens, travaillé dans les grands fonds du Pacifique, sur le mythique Marion Dufresne, le bateau des Terres australes et antarctiques françaises... D'ailleurs, c'est bien lors d'une de ses fameuses missions, qu'un bel officier a gagné son cœur.

Claire, est-ce que je t'ai raconté cette fois où Chantal a remis ses bottes sur le Marion Dufresne ? Cette fameuse mission "Aliénor" en 2004 (figure 4), qui nous a transportées de Lisbonne jusqu'au fin fond de l'Écosse, pour retrouver notre équipe de plongeurs dans le Loch Sunart et les inviter à manger sur le Marion Dufresne, et enfin nous débarquer 2 semaines plus tard à El Ferreol en Espagne... Une mission incroyable où Chantal, avec une volonté de fer, bien qu'étant particulièrement malmenée par le mal de mer a travaillé pendant tous ses quarts - ceux de 8 à 12 - coûte que coûte ... Sauvée par les repas 'chez les malgaches', au pont $\mathrm{C}$, elle a repris ses forces mais s'est brusquement retrouvée victime d'hallucinations curieuses...Elle voyait passer devant son hublot, situé au ras de l'eau ... des listings de papier! Mais elle ne rêvait pas, les hommes de la passerelle les triaient et jetaient à la mer... Plus personne n'oserait faire cela de nos jours ! Cela nous a alors donné des idées, et bientôt Chantal est remontée en nous disant que curieusement elle venait de voir passer une chaussure devant son hublot...Que de crises de fous rires avec Anne-Véronique et Chantal pendant ce quart là et ceux qui ont suivis... Lorsque nous avons débarqué, trois semaines plus tard au port de Ferreol, elle m'a offert un porteclefs avec une chaussure bleue... Un autre souvenir qui me fait toujours sourire. 


\section{XVI'̀mes Journées Nationales Génie Côtier - Génie Civil \\ Le Havre 2020}
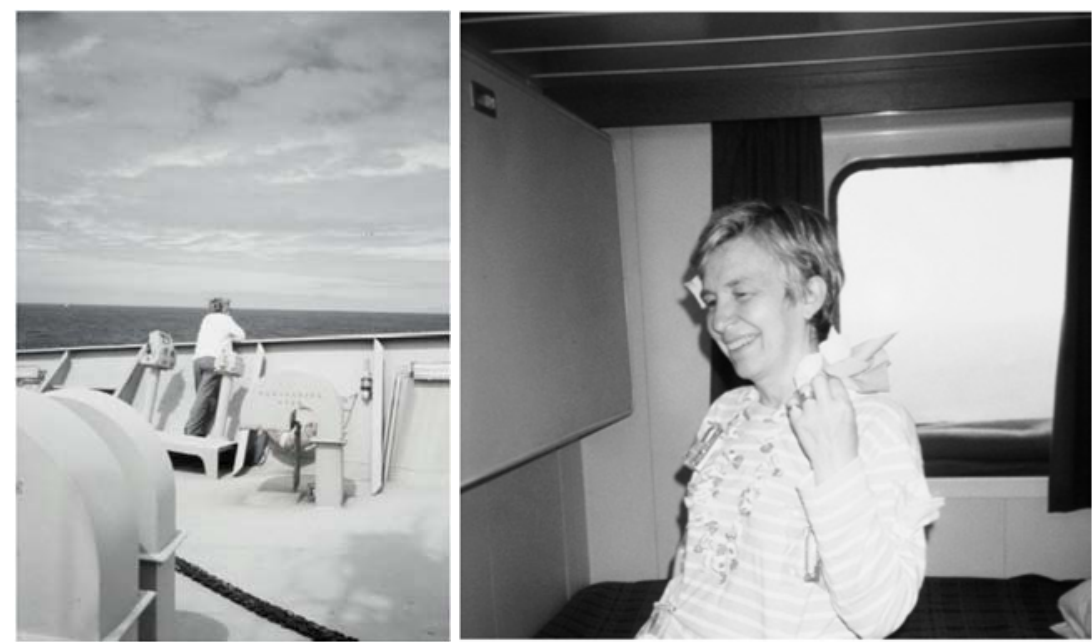

Figure 4. Mission Aliénor, juillet 2004, Lisbonne/El Ferreol, (Photos A.V. Walter-Simonnet).

L'une des grandes forces de Chantal résidait dans sa façon d'enseigner aussi bien aux étudiants qu'aux encadrants. Elle nous transmettait son savoir en racontant, en nous faisant observer, si possible sur le terrain (figure 5), puis en argumentant, point par point si nécessaire...Elle nous a ainsi transmis tout un savoir, tout son savoir ... avec cette façon d'enseigner, plus proche, basée sur les échanges de points de vue, toujours ouverte sur les discussions et les arguments nouveaux. Car Chantal mettait un point d'honneur à être une scientifique honnête, en restant toujours objective et simple, sans se placer hiérarchiquement au-dessus de la mêlée.

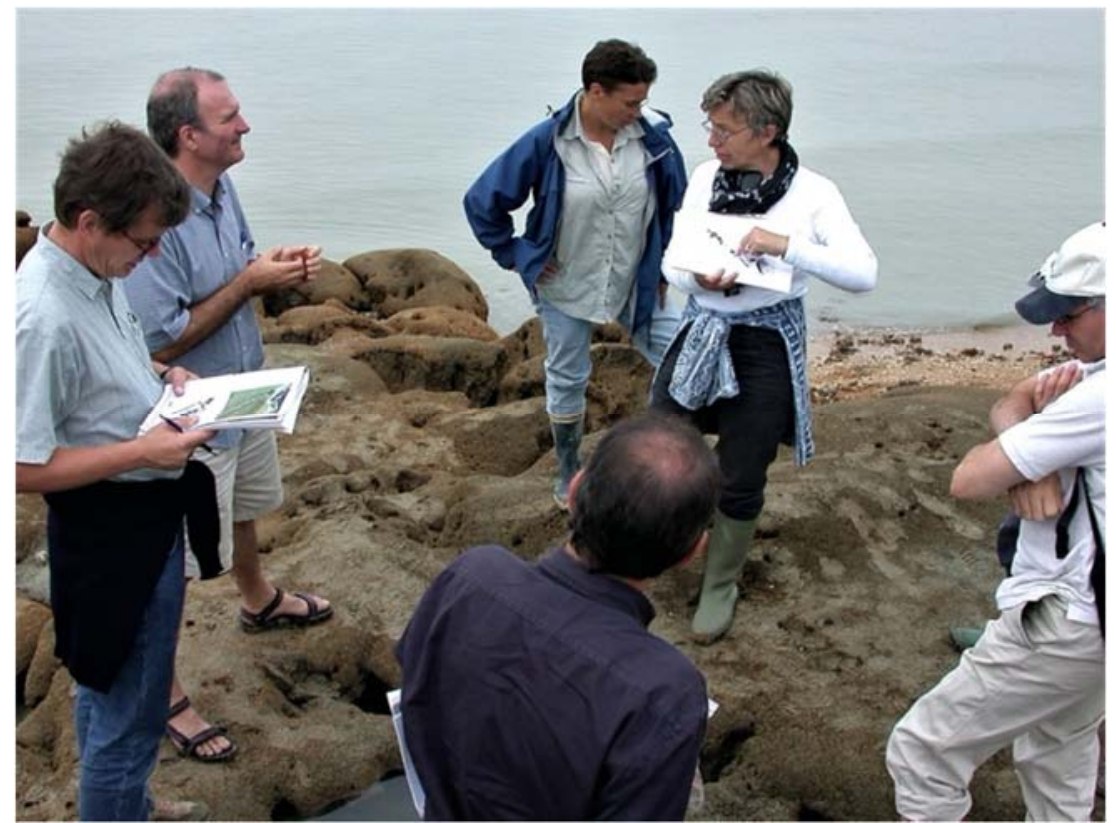

Figure 5. Sortie sur le terrain du site de Champeaux en 2002, (Photo Michael Fuchs). 


\section{Lecture spéciale}

\subsection{Témoignage de Bruno}

\section{Chantal, une "grande sœur" trop tôt disparue}

$\mathrm{Au}$ cours de la vie, il est des rencontres qui comptent énormément et c'est quand la personne disparait que l'on réalise combien cette rencontre fut essentielle. C'est précisément ce qui s'est passé avec Chantal.

Laurence (qui deviendra ma femme) et moi-même avons eu la chance de rencontrer Chantal lorsque nous étions étudiants à l'université d'Orsay, il y a de cela quarante-cinq ans. Chantal aimait nous rappeler d'ailleurs combien elle avait été bienveillante à notre égard lorsqu'elle nous surveillait pendant les examens d'hydrogéologie car elle compatissait nous voyant "sécher" lamentablement face à la dynamique du "coin salé" dans les estuaires.

Après ce début prometteur, c'est dix ans plus tard, lorsque Chantal et Pierre, son mari, ont eu la bonne idée de quitter Les Ulis pour s'installer à Saint-Briac avec leurs filles Marie-Laure, Gwen et bientôt Florence, que notre complicité dans le cadre du travail et des vacances s'est rapidement transformée en une profonde amitié.

Côté sédimentologie littorale, il faut reconnaître que nous avions le privilège d'avoir comme terrain de jeu deux sites exceptionnels : la baie du Mont Saint Michel et l'estuaire de la Rance.

Claire, Agnès, Monique, Odile, Bernadette, Isabelle, Jérôme, Jacques, Jean-Yves, Jihane, Romain, Michaël, Olivier et Pierre, sans oublier notre regretté Alain, la liste est longue des fidèles collègues et amis de Chantal qui comme moi, ne sont pas prêts d'oublier les merveilleux moments passés à organiser et à animer ensemble les stages en baie du Mont Saint Michel pour les "géoscientistes" de TOTAL.

Quelles que soient les circonstances, elle était quelqu'un sur qui l'on pouvait compter humainement et professionnellement. Elle ne transigeait pas avec la rigueur scientifique et l'honnêteté intellectuelle ce qui reste un exemple précieux. De plus, sa bienveillance et sa gentillesse faisaient qu'on se sentait tout de suite bien en sa compagnie.

Chantal avait aussi le sens de la fête ! Un moment mémorable restera le mariage de sa fille Gwénaëlle. Chantal avait eu la bonne idée de regrouper ses amis professionnels autour d'une même table qu'elle avait nommée "la table des scientifiques". Cette table, devenant de plus en plus bruyante au cours de la soirée, les autres convives se souviennent encore aujourd'hui que la science n'est surtout pas synonyme de morosité et d'ennui mais bien au contraire d'humour, de rire et de joie de vivre !

Sa façon d'affronter la maladie en gardant son humour et sa générosité habituels tout en faisant preuve d'une grande sérénité face à l'échec des traitements médicaux est aussi une belle leçon.

Vous comprendrez donc pourquoi, même si la maladie en a décidé autrement, Chantal qui me considérait, sans jamais me l'avouer, un peu comme son "petit frère", est loin de m'avoir quitté et restera à mes côtés pour longtemps encore. 


\section{XVİ̀mes Journées Nationales Génie Côtier - Génie Civil \\ Le Havre 2020}

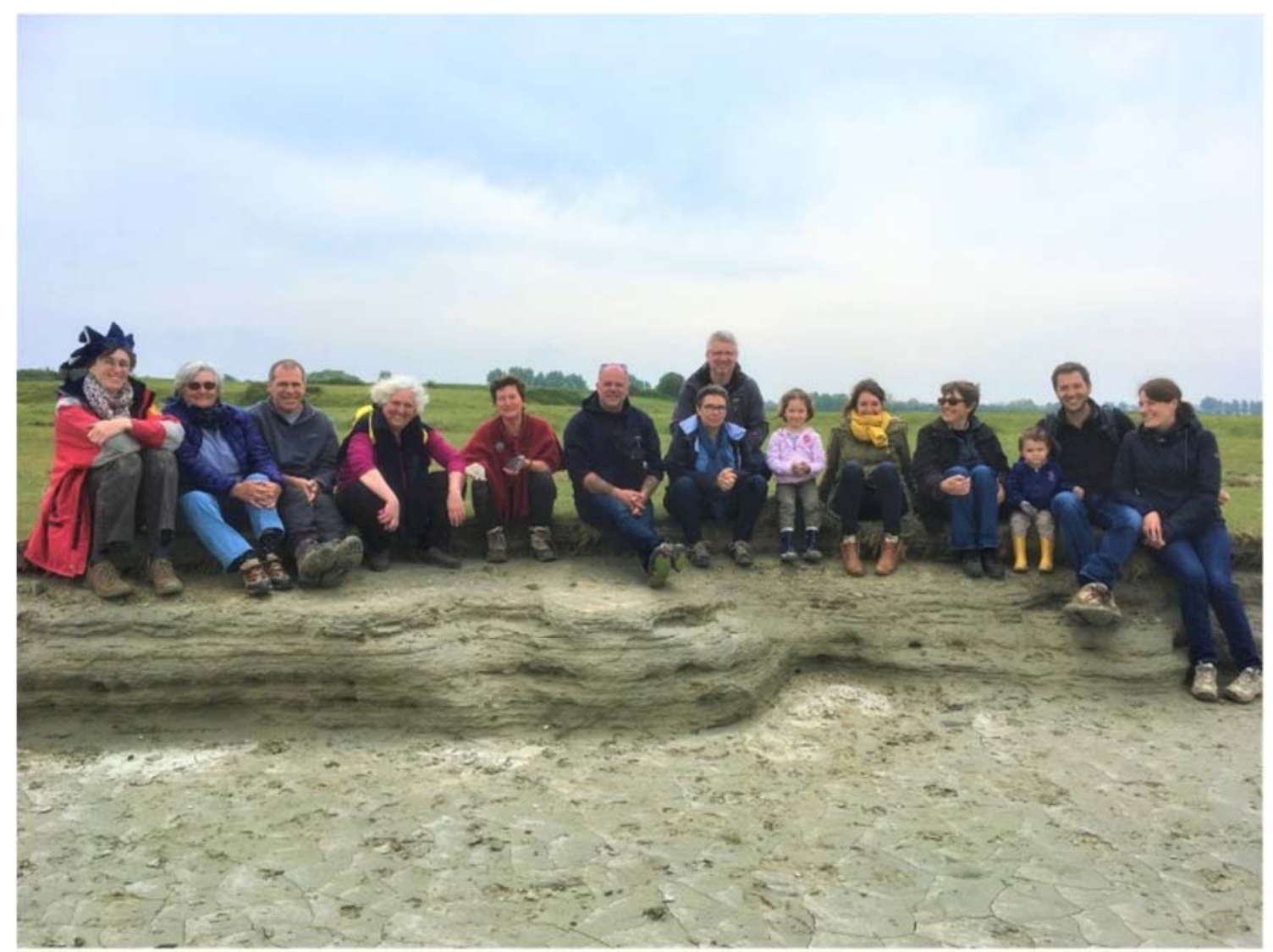

Figure 6. Sur les bords de la Sélune, lors d'une balade à la mémoire de Chantal (2019), pause entre amis au sommet d'une microfalaise de tangue, (Photo Bruno Caline).

Nous avons ainsi perdu non seulement une précieuse collègue mais également une grande amie que nous chercherons encore souvent du regard dans les congrès de sédimentologie, une Grande Dame à laquelle nous pensons régulièrement, naturellement, chaque fois que nous observons un fond d'estuaire, une plage, un littoral, la mer... ou bien encore lorsque nous nous retrouvons pour lui rendre hommage (figure 6).

\section{Merci Chantal!}

\section{Quelques références bibliographiques}

BIZIEN-JAGLIN C. (2002). Les sites et gisements archéologiques du marais de Dol. In Bonnot-Courtois C., Caline B., L'Homer A. Le Vot M. éd., La baie du Mont- SaintMichel et l'estuaire de la Rance. Environnements sédimentaires, aménagements et évolution récente. Bull. Cent. Rech. Elf-Explor. Prod., Mémoire 26, Total Fina ElfCNRS-EPHE, pp 164-176.

BONNOT-COURTOIS C., LEVASSEUR J.-E. (2000). Etudes en Environnement. Volume 5. Contribution à la connaissance de la dynamique des herbus. Final Report of the Joint Initiative for Restoring the Marine Character of Mont-Saint-Michel. Mission 


\section{Lecture spéciale}

Mont-Saint-Michel, Manche Department Infrastructure Directorate (DDE). Univ. Rennes 1, CRI 8586 Prodig CNRS. $196 \mathrm{p}$

BONNOT-COURTOIS C., CALINE B., L'HOMER A., LE VOT M. (2002). La baie du Mont-Saint-Michel et l'estuaire de la Rance. Environnements sédimentaires, aménagements et évolution récente. [The bay of Mont-Saint-Michel and the Rance estuary. Recent development and evolution of depositional environments]. Bull. Cent. Rech. Elf-Explor. Prod., Mémoire 26, Total Fina Elf-CNRS-EPHE, 256 p.

BONNOT-COURTOIS C., BASSOULLET P., TESSIER B., CAYOCCA F., LE HIR P., BALTZER A. (2008). Remaniements sédimentaires superficiels sur l'estran occidental de la baie du Mont-Saint-Michel. European Journal of Environmental and Civil Engineering, vol. 12, $\mathrm{n}^{\circ} 1-2$, pp 51-65. https://doi.org/10.3166/ejece.12.51-65

BONNOT-COURTOIS C., MAZE J.P., LE VOT M., AUGRIS C., EHRHOLD A., SIMPLET L., BLANCHARD M. (2009). Carte morpho-sédimentaire de la baie du Mont Saint-Michel (Ille-et-Vilaine \& Manche), échelle 1:25,000, éd. Quae - Ifremer BONNOT-COURTOIS C., en hommage à A. L'HOMER $(\dagger)$. (2012). Dynamique sédimentaire intertidale en baie du Mont Saint-Michel entre évolution naturelle et aménagements. XII ème Journées Nationales Génie Civil Génie Côtier, Cherbourg, pp 187-222. https://doi.org/10.5150/jngcgc.2012.021-B

BONNOT-COURTOIS C., WALTER-SIMONNET A.-V., BALTZER A. (2014). The Mont-Saint-Michel Bay: An exceptional megatidal environment influenced by natural evolution and man-made modifications. In: Fort M., André MF. (eds) Landscapes and Landforms of France, World Geomorphological Landscapes, Springer, Dordrecht, pp 41-51. https://doi.org/10.1007/978-94-007-7022-5_5

L'HOMER A., COURBOULEIX S., CHANTRAINE J., DEROIN J.P. (1999), avec la collaboration de BONNOT-COURTOIS C., CALINE B., EHRHOLD A., LAUTRIDOU J.-P., MORZADEC-KERFOURN M.T. (1999). Carte géologique de France (1/50 000), feuille Baie du Mont-Saint-Michel, (208), Orléans, BRGM. Notice explicative. 183 p.

L'HOMER A. (2002). Impact sédimentologique des aménagements sur l'estuaire du Couesnon. In Bonnot-Courtois C., Caline B., L'Homer A. Le Vot M. (éd.) La baie du Mont- Saint-Michel et l'estuaire de la Rance. Environnements sédimentaires, aménagements et évolution récente. Bull. Cent. Rech. Elf-Explor. Prod., Mémoire 26, Total Fina Elf-CNRS-EPHE, pp 153-163.

L'HOMER A., BONNOT-COURTOIS C., CALINE B. (2002). Le prisme sédimentaire des dépôts du marais de Dol : essai de reconstitution de la mise en place des dépôts. In Bonnot-Courtois C., Caline B., L'Homer A., Le Vot M. (éd.), La baie du Mont-SaintMichel et l'estuaire de la Rance. Environnements sédimentaires, aménagements et évolution récente. Bull. Cent. Rech. Elf-Explor. Prod., Mémoire 26, Total Fina ElfCNRS-EPHE, pp 177-196. 


\section{XVIèmes Journées Nationales Génie Côtier - Génie Civil \\ Le Havre 2020}

TESSIER B., BILLEAUD I., BONNOT-COURTOIS C., CALINE B. (2007). La baie du Mont-Saint-Michel. Faciès, séquence, évolution. $11^{\text {ème }}$ Congrès français de sédimentologie, Caen 2007, Livret d'excursion, Publ. ASF, n 58, 48 p.

TESSIER B., BONNOT-COURTOIS C., BILlEAUD I., WEILL P., CALINE B. FURGEROT L. (2012). The Mont Saint-Michel bay (NW France). Facies, sequence and evolution of a macrotidal embayment and estuarine environment. TIDALITES 2012, $8^{\text {th }}$ international conference on tidal environments. Field Trip Book Publ. ASF, n 72. 46 p. WEILL P., TESSIER B., MOUAZÉ D., BONNOT-COURTOIS C., NORGEOT C. (2010). Shelly cheniers on a macrotidal flat (Mont-Saint-Michel bay, France) - Internal architecture revealed by ground-penetrating radar. Journal of Sedimentary Geology, Vol. 17. https://doi.org/10.1016/j.sedgeo.2010.12.002 
Lecture spéciale 